\title{
Discourse communities, genres and English as an international language
}

\author{
JOHN SWALES*
}

\begin{abstract}
The concept of discourse community is proposed as a more functional and goal-directed grouping than either speech community or speech fellowship. Six criteria for the existence of a discourse community are outlined and then exemplified. Two claims are advanced: (1) genres are properties of discourse communities, and (2) strong levels of interpersonal relationship are not criterial for the creation of a discourse community. The second half of this paper examines, by way of application of the theoretical discussion, the genre of the reprint request, and discusses the discourse communities that either do or do not participate in the genre. It is argued that the reprint request possesses the full range of genre characteristics, and that the particular source of reprint request cards provides a different kind of evidence about the current status of English as as international language. Finally, the paucity of requests from the Third World is noted, and suggestions made with regard to consequences and remedies.
\end{abstract}

\section{INTRODUCTION}

In this paper I propose a particular conceptualization of the term discourse community and then examine that concept's potential for developing insight into the relationships between texts, text-roles and text-environments in the modern professional world. I will suggest, in particular, that genres are the properties of discourse communities. I next offer, as illustration, a partial analysis of a single genre and, finally, try to show how the results from that analysis have implications both for our understanding of a particular aspect English as an international language (EIL) and for our attempts to make EIL a more equitable instrument of international communication (Labov, 1966).

A second way of looking at speech communities has been to look for patterned regularities in the use of speech. Hence a discourse community is composed of those who share functional rules that determine the appropriacy of utterances. A third way (popular with sociologists and anthropologists) is to look beyond language and claim that a speech community consists of those who share an underlying culture knowledge-value and belief systems and the like. Finally, there are integrationists led by Hymes (1971) who adopt all three criteria: shared linguistic forms, shared regulative rules and shared cultural concepts.

The boundaries of speech communities identified in this way are inevitably more constrained, and such communities come close to Kachru's (1985: 16) concept of speech fellowships:

What we see here, then, is that non-native English-using speech fellowships are using Englishes of the world in their divergent situations and contexts and with various linguistic and ethnic attitudes (original emphases).

An immediate question that arises is whether discourse community is just another name for speech fellowship. I believe there are good reasons for separating the two concepts. First, a sociolinguistic construct like speech fellowship is primarily held together by speech and only secondarily by local or regional writings. In contrast, a sociorhetorical construct like discourse community needs to be neutral in terms of medium and unconstrained by space and time. Members may correspond with other members in distant places; they may

*The English Language Institute, University of Michigan, Ann Arbor, MI 48109, USA. 
react and respond to writings from the past. In consequence, discourse communities are not necessarily neighborly or parochial. Second, the sociolinguistic construct has rather too much of 'community' or 'fellowship' and not enough of 'discourse' for my present purpose. In some obscure but powerful way, in a speech community, the community creates the discourse, while in a discourse community, the discourse creates the community. Certainly, unless we want to extend the shared linguistic forms to include sharing of particular registral features, unless we want to extend shared regulative rules to situations where Gricean maxims do not apply (as in unequal encounters), and unless we want to disperse shared cultural concepts into a wide and often conflicting array of occupational, professional and activity norms, then I do not think the two types of community can be usefully conflated. In terms of the fabric of society, speech communities are centripetal (they pull people in), whilst discourse communities are centrifugal (they set people, or parts of people, apart). To borrow a term from the TESOL organization, an architypical discourse community tends to be a Special Interest Group.

\section{CHARACTERISTICS OF DISCOURSE COMMUNITIES}

In a 1986 conference paper entitled 'The politics of discourse communities', Herzburg (1986: 1) made the following opening observations:

Use of the term 'discourse community' testifies to the increasingly common assumption that discourse operates within conventions defined by communities, be they academic disciplines or social groups.

The idea of a 'discourse community' is not well-defined as yet, but like many imperfectly defined terms, it is suggestive, the center of a set of ideas rather than the sign of a settled notion.

While the accuracy of these observations is not in doubt, it would seem that if discourse community is to be 'the center of a set of ideas' then we can reasonably expect it to be rather more of 'a settled notion' than is apparently the case. I therefore suggest the following defining characteristics of discourse communities:

(a) The discourse community has a communality of interest; i.e. at some level the members share common public goals. The goals are public; spies join discourse communities for private purposes of subversion; people may seek membership in sporting clubs with more or less disguised commercial or sexual intentions. The common public goal may be not that apparent on the surface level. Suppose, for example, there exists a discourse community of legislators, their aides, lobbyists, political journalists etc. As we know, this community will consist of overtly adversarial subgroups, but they all will share some goal such as manufacturing legislation.

(b) The discourse community has mechanisms for intercommunication between members; in terms of Herrington (1985) it will have 'a forum'. The participatory mechanisms may be various: meetings, telecommunications, correspondence, bulletins and so forth.

(c) In consequence of (a) and (b) the discourse community survives by providing information and feedback, even if that information is itself used for various purposes, such as improving performance in a football squad or in an orchestra, making money in a brokerage house, or denting the research front in an academic department.

(d) The discourse community has developed and continues to develop discoursal expectations. These may involve appropriacy of topics, the form, function and positioning of discoursal elements, and the roles texts play in the operation of the discourse community. In so far as "genres are how things get done, when language is used to accomplish them" (Martin, 1985), these discoursal expectations create the genres that articulate the operations of the discourse community (Swales, 1985).

(e) As a result of all of the above, the discourse community possesses an inbuilt dynamic towards an increasingly shared and specialized terminology. Nowhere is this more evident than in the development of community-specific acronyms and abbreviations. It may also develop a tradition of 'in-jokes' and so forth. 
(f) The discourse community has a critical mass of members with a suitable degree of relevant discoursal and content expertise. Discourse communities have changing memberships; people enter as apprentices and leave, by death or in other less involuntary ways. However, survival of the community depends on a reasonable ratio between experts and novices. This is why academic classes are rarely at the outset discourse communities but may develop into them (Marenghi, 1987).

Characterizing discourse communities in this kind of way leads to a number of consequences. First, the fact that groups have things in common in no way implies that such groups form discourse communities. For one reason or another, the following fail to qualify: the stockholders of General Motors, The Book of the Month Club, members of political parties, employees of a major organization, patrons of Harry's Bar, and so forth.

Secondly, sketching the boundaries of discourse communities in ways that $I$ have attempted implies: (a) that individuals may belong to several discourse communities, and (b) that individuals will vary in the number of discourse communities they belong to and hence in the number of genres they command. At one extreme there may be a sense of discourse community deprivation-'Cooped up in the house with the children all day'. At the other extreme, there stands the skilled professional journalists with their chameleonlike ability to assume temporary membership of a wide range of discourse communities.

Third, the criteria I have given do not impose preconditions with regard to at least three features. First, there is no prior expectation of a high level of personal involvement among the members. As we shall see, the discourse community can operate successfully even when the level of personal relationship remains low. Second, a discourse community may or may not be connected to what the members perceive as the central activities and concerns of their lives, activities that are perhaps involved with income, success, family responsibility and so on. Finally, discourse communities will vary in the extent to which they are normdeveloped, or have their set and settled ways. Some will be extremely conservative ('This is the way we do things here') while others may be constantly evolving.

This concept of discourse community still has a number of unresolved problems. One of these was described by Hazem Najjar, a graduate student, as the 'Cafe Owner' problem. In generalized form, the problem goes as follows: Individuals A, B, C and so on occupy the same professional roles in life. They interact (in speech and writing) with the same clienteles; they originate, receive and respond to the same kind of messages for the same purposes; they have an approximately similar range of genre skills. And yet, as cafe-owners working long hours in their own establishments, and not being members of the Local Chamber of Commerce, A, B and C never interact with one another. Do they form a discourse community? Or to put it another way, what do we do about 'visible uncolleges', in which there is no apparent 'forum'? Notice that the 'Cafe Owner' problem is not quite like those situations where A, B and C operate as 'points'. A, B and C may be lighthouse keepers on their lonely rocks, or missionaries in their separate jungles, or neglected consular of ficials in their rotting outposts. In all these cases, although A, B and C may never interact, they all have lines of communication back to base, and presumably acquired discourse community membership as a key element in their initial training. Bizzell (1987:5) argues that this kind of social group will be a discourse community because "its members may share the social-class-based or ethnically-based discursive practices of people who are likely to become cafe-owners in their neighborhood". However, even if this sharing of discursive practice occurs, it does little to resolve the logical problem of assigning membership of a community to individuals who neither admit nor recognize that such a community exists. 


\section{DISCOURSE COMMUNITIES IN ACTION}

Recently there have been questions raised as to whether a hobby-group could constitute a discourse community. I believe, in the light of my criteria and on the basis of personal experience, that the answer is firmly in the affirmative. As it happens, I belong to two hobby groups. One is a world-wide philatelic grouping of about 300 people who specialize in the postal history of Hong Kong. This is a group that operates partly by correspondence and phone call, but principally through a bi-monthly bulletin and newsletter (the latest to arrive is No. 260). Two extracts here follow from the bulletin and an illustrative lot from a recent specialized auction catalogue.

\section{Bulletin extracts}

2. Hong Kong, Type 12 , with index

No one has yet produced another example of this c.d.s. that I mentioned on J. 256/7 as having been found with an index letter ' $C$ ' with its opening facing downwards, but Mr. Scamp reports that he has seen one illustrated in an auction catalogue having a normal ' $C$ ' and dated MY 9/59 (Type 12 is the 20mm singlecircle broken in upper half by HONG-KONG). It must be in someone's collection!

3. The B.P.O.'s in Kobe and Nagasaki

Mr. Pullan disputes the statement at the top of J. 257/3 that 'If the postal clerk had not violated regulations by affixing the MR 17/79 (HIOGO) datestamp on the front, we might have no example of this c.d.s. at all.' He states that 'By 1879 it was normal practice for the sorter's datestamp to be struck on the front, the change from the back of the cover occurring generally in 1877, though there are isolated earlier examples', thus there was no violation of regulations.

\section{Auction catalogue entry}

11761899 Combination PPC to Europe franked CIP 4 C canc large CANTON dollar chop, pair HK 2 C carmine added \& canc Hong Kong index B cds. Arr cds. (1) (Photo) HK $\$ 1500$.

These three extracts suggest that the Hong Kong Study Circle meets all six of the proposed defining criteria. There is a common goal, forum, information exchange, genre development, specialized terminology and expertise. The catalogue entry is a key subgenre in this discourse community, and as such, it is likely to present processing problems to non-members. Even if luck and skill were to combine to interpret PPC as 'picture postcard', CIP as 'Chinese Imperial Post' and a 'combination' as a postal item legitimately combining the stamps of two or more nations, the outsider would still not be in a position to estimate whether 1500 Hong Kong dollars is an appropriate sum to bid.

The second hobby group I belong to is the local Audubon Society. As a local organization, the forum in this case is created by the telephone, a newsletter, and monthly field-trips, meeting and talks. One interesting aspect of discussion among birdwatchers at the local Audubon is the high level of technical rhetoric displayed and expected (although the very young, the very old and the very new are excused this requirement). Thus we find comments like:

Apart from Blackpolls fall immatures seem down this year.

Or this kind of carefully formulated question raised after the last talk I attended:

Would you like to estimate the relative seriousness of gull versus crow predation of eggs and hatchlings?

Again this feels like a discourse community to me, just as I feel that there is one in the unit in which I work. 
Although the two hobby groups differ in that one is distanced and the other proximate, they share several features. Both are essentially detached from personal involvement; in neither case do I know much about what my co-members do for a living, whether they are rich or poor, are married or single, believe in a supernatural being, etc. Nor do these discourse communities have much to do with a 9-to-5 existence, or with paying the bills or with educational progress. Of course other hobby groups may be different: members of amateur dramatic discourse communities are somewhat notorious for their level of interpersonal involvement, whilst membership of professional associations may be closely connected to the business of a career.

The third example is academic, for I have chosen to apply my emerging thoughts to Anne Herrington's study of the contexts for writing in two college chemical engineering classes (Herrington, 1985). I have done this because I believe the Herrington study to be a highly regarded ethnographic study of a particular academic milieu. Herrington concluded that the Chem. Eng. Lab. course and the Chem. Eng. Design Process course "represented distinct communities where different issues were addressed, different lines of reasoning used, different writer and audience roles assumed, and different social purposes served by writing'" (Herrington, 1985: 331). If we also note that the two courses were taught in the same department at the same institution by the same staff to largely the same students, then the Herrington study suggests additionally that there may be more of invention than we would like to see in our models of disciplinary culture.

However, if we apply the variable features of discourse communities that I have mentioned to the Herrington findings, we can, at the least, obtain another angle on the disparity. Writing in the lab course was connected to the 'display familiarity' macro-act of college assignments (Horowitz, 1986). Writing in the design course was connected to the persuasive reporting macro-act of the outside professional world. The lab course was norm-developed, while the design course was norm-developing. As Herrington observes, in the lab both students and faculty were all too aware that the conceptual issue in the assignments was not an issue for the audience-the professor knew the answers. But it was an issue in Design. As a part consequence, the lab 'forum' was much more disengaged than that in Design, where professor and student interacted together in a joint problem-solving enviroment.

\section{GENRE, DISCOURSE COMMUNITY AND THE REPRINT REQUEST}

A reprint request (RR) is a request for a reprint (or copy) of a publication mailed to that publication's author. Typically, the request is carried by a printed or duplicated card with spaces provided for inserting the name of the author, the details of the publication and the name of the requester. The RR genre is an interesting phenomenon for those with an interest in world Englishes. Some of its interesting features are noted below:

(a) The genre is large, with many millions of RRs mailed out each year (Swales, 1988).

(b) The genre is very unevenly distributed amongst academic fields, disciplines, and discourse communities. In some, such as the life, health and physical sciences, the sending of RRs has become fairly standard practice in certain parts of the world; in others, such as the humanities, it remains very rare. It remains fairly rare in fields like applied linguistics, but is becoming commoner in more quantitative areas such as testing. In my own case, in only one instance have I received more than 20 RRs for a paper; that paper appeared in a journal called Scientometrics, which is devoted to quantitative studies of the history and sociology of science. The fact that I received an abnormal number of requests suggests that by publishing in Scientometrics I was joining a new and different discourse community (Swales, 1986). 
(c) The main trigger for RRs would appear to be abstracting journals like Current Contents, rather than the primary sources (Onuigbo, 1985).

(d) The RR provides unusual information about sociolinguistic patterns of language use in international research. Much of the available evidence derives from higher-order policies, the policies of journals, abstracting services, international scientific and scholarly associations and the like [e.g. Baldauf and Jernudd (1983)], or from studies of the language practices of individual scholars [e.g. St. John (1987)]. However, the fact that RRs are typically printed by a department or a research unit (for completion by individual members) gives us access to language decision making carried out at an intermediate level.

Given my feeling that the RR is, in a number of ways, a not insignificant type of text, I undertook a pilot corpus-based study of the genre. The main corpus consists of 127 printed RRs generated as a result of the publication of three papers, whilst the subsidiary corpus consists of 12 further requests of a different form (four ISI Request-A-Prints and eight more personal requests). The three papers are:

(1) Swales. J. M. (1985) English language papers and authors's first language: preliminary explorations. Scientometrics, 8, 91-101.

(2) Fogel, R. and Trappe, J. M. (1978) Fungus consumption (mycrography) by small animals. Northwest Science, 52, 1-31.

(3) Fogel, R. (1983) Root turnover and productivity of coniferous forests. Plant and Soil, 71, 75-85.

The 1978 Fogel paper was published in Northwest Science, which, as its name implies, is an interdisciplinary regional US journal. The 1983 paper was published in a specialized international journal produced in Britain.

The difference between the role and location of the three journals could be expected to have some effect on the provenance of the requests. Although this is undoubtedly the case with regard to Swales vs Fogel, it is much less obviously true with regard to Fogel 1978 vs Fogel 1983. The figures for provenance (grouped into main areas of the world) are given in Table 1.

Table 1 Provenance (main and supplementary corpus)

\begin{tabular}{|c|c|c|c|c|c|c|c|c|}
\hline & USA & Canada & $\begin{array}{l}\text { Northern and } \\
\text { Western Europe }\end{array}$ & $\begin{array}{l}\text { Eastern Europe } \\
\text { and Russia }\end{array}$ & $\begin{array}{c}\text { Latin } \\
\text { America }\end{array}$ & $\begin{array}{c}\text { Australia/ } \\
\text { Orient }\end{array}$ & Other & Total \\
\hline Swales 1985 & 2 & 1 & 12 & 14 & 5 & 1 & Turkey & 36 \\
\hline Fogel 1978 & 34 & 4 & 4 & 0 & 2 & 2 & 0 & 46 \\
\hline Fogel 1983 & 31 & 6 & 9 & 4 & 1 & 5 & India & 57 \\
\hline Total & 67 & 11 & 25 & 18 & 8 & 8 & 2 & 139 \\
\hline
\end{tabular}

The main origin of requests for the Scientometrics paper is clearly Europe, perhaps because the journal is edited in Eastern Europe, and distributed from Western Europe. However, the pattern of provenance for the two Fogel papers is perhaps less different than we might expect. US provenance clearly predominates in both cases, while proportions for Canada and Latin America/the Pacific Rim are roughly equivalent. Although European requests do rise in the case of the Plant and Soil paper, they still only amount to 13 (in contrast to 37 from North America). In all three cases, RRs from the Third World were few.

The language use patterns in the printed/duplicated cards in the main corpus $(N=127)$ are interesting. The percentage figures for the languages occurring are shown in Table 2. As can be seen, English was ubiquitous; in fact, $80 \%$ of the cards were monolingual English, and English was the only language found on monolingual cards. German and French were commonly employed on the multilingual cards. The six cards carrying a Russian-language request all emanated from the Soviet Union or Eastern Europe. Only one of the 10 cards sent from Hispanic/Portuguese-speaking areas used Spanish, suggesting that Spanish has 
very marginal status as a RR language (the other Spanish card came from a French university). As far as the corpus is concerned, Italian failed to establish itself as an RR language and Japanese and Chinese have yet to do so.

Table 2. Percentage of language occurrence in a sample of RRs

\begin{tabular}{lc}
\hline English & $100 \%$ \\
German & $15 \%$ \\
French & $13 \%$ \\
Russian & $5 \%$ \\
Spanish & $1.5 \%$ \\
\hline
\end{tabular}

The $R R$ has a recognized name within the relevant discourse communities, and members of those communities recognize the RR as being an identifiable communicative act. Those members also share an understanding of what the public purpose of a $R R$ is, and respond to that purpose with a limited set of behaviors. The $R R$ can also function as a standard and typical response-type; in certain disciplines, evidence from both total numbers and relative percentages strongly suggests that an $R R$ is a normal way of obtaining a copy of a potentially interesting article. The remaining issue, therefore, is whether an RR is a sufficiently structured and standardized communicative event (with consequent constraints on allowable contributions in terms of purpose, positioning and form) to constitute a genre.

The central element of the reprint request can be expected to tbe the request itself (plus adequate information to indicate where the request should be sent).

This central element is typically bounded by opening and closing salutations of types that occur throughout the range of formal correspondence genres. Given the central purpose of making a request, it also is not surprising to find that request element is often followed by an anticipatory expression of thanks. This element would seem to be motivated by two considerations. On the one hand requesters may feel that a grateful acknowledgement of compliance will enhance the chances of a positive response. Secondly, the 'thanks' statement occurs on RRs because of the essentially anticipatory 'one-shot' nature of the communicative event. Under normal circumstances, neither requester nor responder expects that the receipt of the requested article will call forth a 'thank-you' note or letter.

In genre-analysis it is often as valuable to observe what is not there as to what actually occurs [cf. Becker (1984) on 'silential relations']. In none of the 127 printed cards in the main corpus is there an example of those anticipatory receipts that are common in many kinds of solicitating or offer letters; nothing of the following kind occurs:

I am looking forward to receiving your paper.

In anticipation of a speedy response, etc.

Presumably, such formulations are contra-indicated because they are somehow seen as 'presumptive' and thus 'heavy' or 'risky'.

The central request itself is the longest and most complex statement in the RR and contains two kinds of optional elements: stylistic options (such as choice of first-person pronoun or the inclusion of adverbs), and semantic options (such as asking for related papers or giving some reason for making the request).

The actual requests (in English) varied in length from six to 41 words. The typical opening was the request itself, and in the corpus examined here, there is striking evidence for a major structure (107 instances) and a number of minor structures (a total of 20 instances). 
Fourteen of the 20 examples of the minor structures involved the lexical item send. The more common variants were:

(Would you) please send me/us ...

(six cases)

I would be very grateful if you could send me/us . . .

(four cases)

It would be greatly appreciated if you could send me ...

(two cases)

However, the much preferred approach in the RR is not to request that a reprint be sent, but rather to express appreciation for a reprint to be received. The linguistic regularity of this major rhetorical structure can be seen in Table 3.

Table 3. The major request form in $\operatorname{RRs}(N=107)$

\begin{tabular}{|c|c|c|c|c|c|}
\hline $\begin{array}{l}\text { I } \\
(102 / 107)\end{array}$ & $\begin{array}{c}\text { would } \\
(99 / 107)\end{array}$ & $\begin{array}{c}\text { greatly } \\
(55 / 107) \\
0 \\
(40 / 107)\end{array}$ & $\begin{array}{c}\text { appreciate } \\
(107 / 107)\end{array}$ & $\begin{array}{c}\text { receiving } \\
(52 / 107) \\
0 \\
(51 / 107)\end{array}$ & a copy/reprint \\
\hline
\end{tabular}

We can see from Table 3 that there are strong convergences for $I$ would and a $100 \%$ choice of the verb appreciate. Two optional elements appear to be the inclusion of an intensifying adverb, most typically 'greatly' and the addition of 'receiving'. I suspect that the very marked preference for appreciating receipt rather than requesting sending is that the former is less likely to conjure up images in the mind of the recipient of the chores that have to be completed in order to get the reprint or photocopy dispatched!

Finally, we can consider four further possibilities that may add to or detract from the rhetorical effectiveness of the request. Although these semantic options vary from very rare to fairly uncommon, they are, nevertheless, worth some discussion.

The first is to make the request ostensibly conditional on availability (perhaps as a device for obviating the unpleasant surprise of being charged). There were just seven of these, all from North America and all in the Fogel corpus. Five preceded the request and two succeeded it:

Pre-request: If available for distribution,

If available for free distribution,

If the following publication is available for distribution,

If a reprint is available,

If copies are available,

Post-request: $\quad$. . if available,

$\ldots$ if copies are available for distribution.

Next, the corpus produced but four instances (all from the USA) of printed statements that referred to the uses to which the RR would be put:

... for current reference and permanent files. (two cases)

... for use in my research program.

... for use in my teaching and research.

Third, there is one instance of an RR that attempts to both justify response and yet empathize with the recipient of the RR. The card (from the Swales corpus) is unique in the amount of 'rhetorical work' it essays and for this reason the long and complex central element is worth quoting in full:

As an institution responsible for providing scientists and oncologists throughout the German Democratic Republic with medical literature we would be exceedingly grateful if you could send us a reprint, or if it should not be available, a photocopy of your article. 
Knowing that such requests can become burdensome we wish to assure you, (sic) that your assistance will be most appreciated.

These optional additions are rare enough to suggest that most producers of RRs probably believe that such embellishments have little effect on response rate. However, where they are found they are clearly designed to increase goodwill.

Any such goodwill may, of course, be diminished by requests that are considered unnecessarily onerous. It is for this reason that tagged-on requests for other papers present a potential problem for RR designers. Twenty-nine out of the 127 cards $(23 \%)$ had these tagged requests, 20 of them coming from outside the USA suggesting that in the USA such tags are seen as either unnecessary or unwarranted or self-defeating. Seventeen of the tags were attached to the request sentence, while 12 were following independent sentences. The use of a sentence boundary can perhaps be seen as creating a separating or distancing effect between the two requests; the use of two sentences was very common among the US cards. Most of the other 17 had forms similar to one of the following:

, and reprints of related papers.

, and any other reprints on similar topics.

, as well as other related publications.

Only one offered any purpose motivation:

, and any other publications which would aid our studies.

Interestingly, nine of the 12 independent sentences showed a switch into the passive, even though they tended to retain appreciate as the verb $(8 / 12)$. Typical examples are:

Copies of other papers on this and similar subjects would be appreciated. (three cases)

Copies of related articles would also be appreciated. (two cases)

\section{CONCLUSIONS AND APPLICATIONS}

The RR has all the characteristics of a well-established genre. It has size and stability, and has, within the discourse communities in which it occurs, a narrowly-defined and mutually understood role as being a 'one-shot' request for a simple response. The request itself is the central illocutionary act of the RR and consists of a number of obligatory elements and a number of optional elements that may be incorporated either for clarification (e.g. 'which appeared in') or designed to enhance the rhetorical effectiveness of the request (e.g. 'if available for distribution'). In addition the specific request may have tagged on to it a broader aspiration (e.g. 'and related papers'). In a clear majority of cases, the RR also contains an opening salutation, an anticipatory expression of thanks and a closing salutation. With the exception of the availability option, the order of these elements is fixed.

RRs are always expressed in English, but in a minority of cases other languages may be used as well. The maximum number of languages used in this corpus was four (five instances). German and French are relatively common, Russian and Spanish rare. If a card is multilingual, English is likely to be the second and not the first language listed. Language choice can be related to the location of the institution that generates the RR and to the linguistic and geo-political circumstances that affect that location.

The RR, despite its intrinsically humble nature, is a prototypic exemplar of EIL: As such it is an established vehicle of communicative traffic in those discourse communities that have come to accept the RR as part of their networking practices. However, data from both the Onuigbo corpus and the present corpus indicate that LDCs produce few reprint 
requests. Even if we take into account limited access to journals and abstracts in the Third World, the low figures are surprising for at least two reasons. First, the printing and mailing of RRs is a much cheaper way of obtaining published papers than any other-and there is evidence that response rates to RRs are reasonably high (Swales, 1986). Second, we have some evidence (although much of it is anecdotal) that many non-native speakers, being diffident about their English-writing abilities and also anxious not to appear obtrusive to distant scholars, find it difficult to initiate academic correspondence with potential colleagues in other parts of the world. Those of us who feel concern about the isolation of these groups in a time of increasing predominance of English can encourage attempts to diminish disenfranchisement by advocating the production and use of RRs. To some this may seem a trivial recommendation, but if, by means of RRs, isolated non-native speakers of English establish and begin to develop the contacts they so badly need, then the outcome may not be trivial at all. Certainly, any developments along these lines would be consistent with others in the area of pragmatics and EIL.

\section{REFERENCES}

Baldauf, Richard B. and Jernudd, Bjorn H. (1983) Language use patterns in the fisheries periodical literature. Scientometrics, 5, 245-255.

Bizzell, Patricia (1987) Some uses of the concept of "discourse community". Paper presented at the Pennsylvania State Conference on Composition, July 1987.

Becker, Alton L. (1984). Biography of a sentence: a Burmese proverb. In Text, Play and Story: The Construction and Reconstruction of Self and Society. Edited by E. M. Bruner. Washington, DC: American Ethnological Society. pp. 135-155.

Braithwaite, Charles A. (1985) Towards a conceptualisation of "speech community". In Papers from the Minnesota Regional Conference on Language and Linguistics. Edited by N. Stenson. University of Minnesota. pp. 13-29.

Herrington, Anne (1985) Writing in academic settings: a study of the contexts for writing in two college chemical engineering courses. Research in the Teaching of English, 19, 331-361.

Herzburg, Bruce (1986) The politics of discourse communities. Paper presented at the College Composition and Communication Convention, New Orleans, March 1986.

Horowitz, Daniel M. (1986) What professors actually require: academic tasks for the ESL classroom. TESOL Quarterly, 20, 445-462.

Hymes, Dell (1971) Sociolinguistics and the ethnography of communication. In Social Anthropology and Language. Edited by E. Ardner. London: Tavistock. pp. 47-93.

Kachru, Braj B. (1985) Standards, codification and sociolinguistics realism: the English language in the Outer Circle. In English in the World. Edited by Randolph Quirk and H. G. Widdowson. Cambridge: Cambridge University Press. pp. 11-30.

Labov, William (1966) The Social Stratification of English in New York City. Washington, DC: Center for Applied Linguistics.

Marenghi, Eleanor (1986) Classroom research: questions from the audience in a university lecture course. Unpublished Ph.D. dissertation, University of Michigan, Ann Arbor, MI.

Martin, J. R. (1985) Process and text: two aspects of human semiosis. In Systemic Perspectives on Discourse I. Edited by J. D. Benson and W. S. Greaves. Norwood, NJ: Ablex. pp. 246-274.

Onuigbo, Wilson I. B. (1985) Reprint requests-a tool for documentation. International Forum for Information and Documentation, 10, 7-9.

St. John, Maggie-Jo (1987) Writing processes of Spanish scientists publishing in English. English for Specific Purposes, 6, 113-120.

Swales, John M. (1985) A genre-based approach to language across the curriculum. In Language Across the Curriculum. Edited by Makhan L. Tickoo. Singapore: RELC. pp. 10-22.

Swales, John M. (1986) ESP in the big world of reprint requests. English for Specific Purposes, 5, 81-86.

Swales, John M. (1988) Language and scientific communication: the case of the reprint request. Scientometrics, $13,93-101$. 